OPEN ACCESS

Edited by:

Lin Lin,

Jiangsu University, China

Reviewed by:

Xucong $L V$,

Fuzhou University, China

Mao-Ke Liu,

Sichuan Academy of Agricultural

Sciences, China

*Correspondence:

Zheng-Hong $X_{L}$

zhenghxu@jjiangnan.edu.cn

Specialty section: This article was submitted to

Food Microbiology,

a section of the journal

Frontiers in Microbiology

Received: 30 April 2019

Accepted: 04 June 2019

Published: 21 June 2019

Citation:

Chai L-J, Lu Z-M, Zhang X-J, Ma J, Xu P-X, Qian W, Xiao C, Wang S-T,

Shen C-H, Shi J-S and Xu Z-H (2019)

Zooming in on Butyrate-Producing

Clostridial Consortia in the Fermented

Grains of Baijiu via Gene Sequence-

Guided Microbial Isolation.

Front. Microbiol. 10:1397.

doi: 10.3389/fmicb.2019.01397

\section{Zooming in on Butyrate-Producing Clostridial Consortia in the Fermented Grains of Baijiu via Gene Sequence-Guided Microbial Isolation}

\author{
Li-Juan Chai ${ }^{1,4}$, Zhen-Ming Lu ${ }^{1,4,5}$, Xiao-Juan Zhang ${ }^{1,4,5}$, Jian $\mathrm{Ma}^{2}$, Peng-Xiang $X \mathrm{U}^{2}$, \\ Wei Qian' ${ }^{2}$, Chen Xiao' ${ }^{2}$, Song-Tao Wang ${ }^{5}$, Cai-Hong Shen ${ }^{5}$, Jin-Song Shi ${ }^{3}$ and \\ Zheng-Hong $X u^{1,2,5 *}$
}

${ }^{1}$ National Engineering Laboratory for Cereal Fermentation Technology, Jiangnan University, Wuxi, China, ${ }^{2}$ Key Laboratory of Industrial Biotechnology of Ministry of Education, School of Biotechnology, Jiangnan University, Wuxi, China, ${ }^{3}$ School of Pharmaceutical Science, Jiangnan University, Wuxi, China, ${ }^{4}$ Jiangsu Engineering Research Center for Bioactive Products Processing Technology, Jiangnan University, Wuxi, China, ${ }^{5}$ National Engineering Research Center of Solid-State Brewing, Luzhou, China

Butyrate, one of the key aroma compounds in Luzhou-flavor baijiu, is synthesized through two alternative pathways: butyrate kinase (buk) and butyryl-CoA: acetate CoA-transferase (but). A lack of knowledge of butyrate-producing microorganisms hinders our ability to understand the flavor formation mechanism of baijiu. Here, temporal dynamics of microbial metabolic profiling in fermented grains (FG) was explored via PICRUSt based on $16 \mathrm{~S}$ rRNA gene sequences. We found Bacilli and Bacteroidia were the major potential butyrate producers in buk pathway at the beginning of fermentation, while later Clostridia dominated the two pathways. Clone library analysis also revealed that Clostridia ( $73 \%$ OTUs) was predominant in buk pathway throughout fermentation, followed by Bacilli and Bacteroidia, and but pathway was merely possessed by Clostridia. Afterward, Clostridia-specific 16S rRNA gene sequencing demonstrated Clostridium might be the major butyrate-producing genus in two pathways, which was subsequently evaluated using culture approach. Seventeen Clostridium species were isolated from FG based on 16S rRNA gene sequenceguided medium prediction method. Profiles of short-chain fatty acids and but and buk genes in these species demonstrated phylogenetic and functional diversities of butyrateproducing Clostridium in FG. These findings add to illustrate the diversity of potential butyrate producers during brewing and provide a workflow for targeting functional microbes in complex microbial community.

Keywords: butyric acid, baijiu fermented grains, complex microbial community, Clostridia, Clostridium isolation

\section{INTRODUCTION}

Luzhou-flavor baijiu is a kind of Chinese distilled spirits produced from different grains (mainly sorghum) by spontaneous solid-state fermentation in a mud pit, and it accounts for more than $70 \%$ of Chinese baijiu production (Tao et al., 2014). The production process of Luzhouflavor baijiu can be generally divided into three phases: Daqu (fermentation starter) making, 
in-cellar fermentation, and distillation (Zheng and Han, 2016). During the in-cellar fermentation stage, Daqu, steamed grains, and rice husk are mixed and sealed in a fermentation pit for around 40-60 days. The mixed grains in fermentation pit provide the habitat and substrate for the proliferation and metabolism of microorganisms during brewing (Wang et al., 2008). A variety of flavor compounds are formed in fermented grains through the metabolism of multiple brewing microorganisms (Wang et al., 2014), determining the typical and unique flavor of Luzhou-flavor baijiu (Fang et al., 2019), although it is mainly constituted of ethanol $(38-65 \%, v / v)$ and water (Zheng and Han, 2016). The representative aroma compounds of Luzhou-flavor baijiu are predominantly ethyl hexanoate, ethyl butanoate, ethyl acetate, ethyl lactate and their corresponding acids, hexanoic acid, butanoic acid, acetic acid, and lactic acid (Fan and Qian, 2006; Liu and Sun, 2018; Fang et al., 2019). The aroma intensity characterized by Osme values of butanoic acid (rancid and cheesy aroma, Osme value $=15$ ) and its main derivative ethyl butanoate (pineapple aroma, Osme value $\geq 14$ ) almost ranked first among the volatile compounds according to Fan and Qian (2006). Thus, shedding light on the butyrate-producing microbial consortia in fermented grains is conductive to elucidate the flavor formation mechanism of Luzhou-flavor baijiu.

Culture-independent approaches (e.g., DGGE, 16S rRNA sequencing) revealed that Bacilli, Bacteroidetes, and Clostridia dominated the bacterial consortia in fermentation grains (Wang et al., 2008), either during brewing (Wang et al., 2017) or from different-aged fermentation cellars (Ding et al., 2015). At the genus level, Lactobacillus was predominant, particularly at the later stage of fermentation (Wang et al., 2017). Using culture method, most of the isolated bacteria were scattered in Bacillus, Lactobacillus, and Acetobacter (Zou et al., 2018b). By performing transcriptome analysis, synergistic effect was identified between Lactobacillus and Saccharomyces in the sulfur compound production (Liu et al., 2017). Moreover, Lactobacillus and Bacillus were found as dominant producers of lactic acid and other organic acids. However, as one of the key aroma compounds in Luzhou-flavor baijiu, taxonomic distribution of butyrate-producing microorganisms in fermentation grains during brewing remains unclear to date.

For the final step of microbial butyrate synthesis from butyryl-CoA, there are two alternative pathways namely butyrate kinase (buk) pathway and butyryl-CoA:acetate CoA-transferase (but) pathway (Figure 1A). The key genes but and buk were previously chosen to identify these two pathways (Louis et al., 2010; Vital et al., 2014, 2015). In anaerobic environments, butyrate could be synthesized by butyrate-producing bacteria related to Eubacterium spp. and Roseburia spp. (Clostridium cluster XIVa) and Faecalibacterium prausnitzii (Clostridium cluster IV) (Louis and Flint, 2009, 2017). The fermentation of Luzhou-flavor baijiu was also carried out under an anaerobic environment. Metagenomic analysis showed that Clostridium was most likely involved in butyrate production through buk pathway in pit mud (Tao et al., 2017). However, most of Clostridial microbes are difficult to be isolated and cultivated owing to their oxygen sensitivity. Thus, obtaining natural strains with high-yielding butyrate potential is challenging for researchers.

In the previous work, Clostridia class was identified as the predominant putative butyrate producers in both buk and but pathways in pit mud (i.e., fermentation container) of Luzhouflavor baijiu and the butyrate-producing potential of some Clostridium strains were evaluated (Chai et al., 2019). To further unravel the source of butyrate in baijiu brewing ecosystem, the present study aimed to investigate the butyrate-producing bacterial consortia in baijiu fermented grains (i.e., fermentation substrate). Temporal distribution of functional genes involved

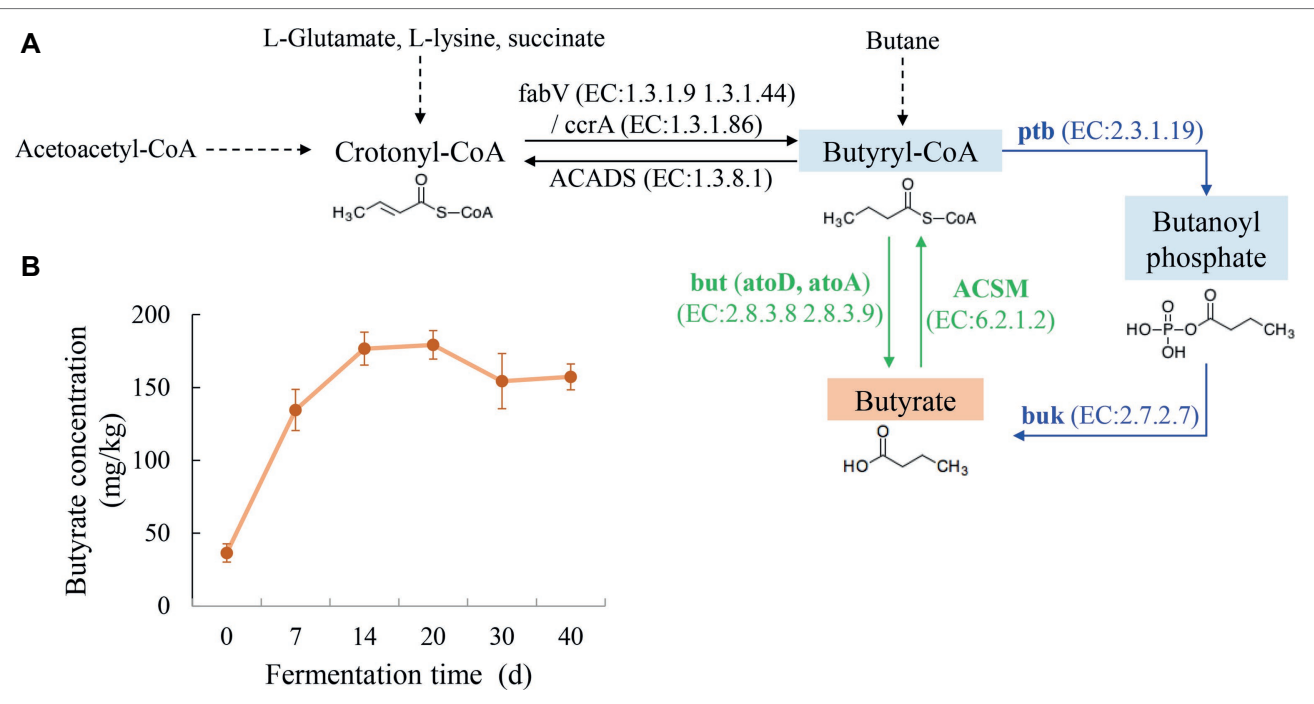

FIGURE 1 | (A) Putative microbial butyrate metabolism pathway in the fermented grains of Luzhou-flavor baijiu based on the information from KEGG database (ko00650). The enzyme IDs in KEGG were shown in brackets. Key enzymes involved in but / buk pathway were highlighted by green/blue color. (B) Butyrate content in fermented grains during brewing. 
in the conversion between butyryl-CoA and butyrate was firstly illustrated. The potential butyrate-producers were further investigated via the analysis of buk and but genes' phylogenetic diversities. Subsequently, the phylogenetic diversity and pathway distribution of the major butyrate-producing group, Clostridia, was conducted. The major potential butyrate-producing Clostridium was isolated and their butyrate-producing capacity was assessed. This work is expected to elucidate the main potential butyrate-producing bacterial community in fermented grains during baijiu brewing.

\section{MATERIALS AND METHODS}

\section{Sampling and Storage}

Fermented grains used in this study were collected from the fermentation pit of 30 years old (length $\times$ width $\times$ height $=4.3 \mathrm{~m} \times 3.3 \mathrm{~m} \times 2.3 \mathrm{~m})$ in Luzhou Laojiao Group Co., Ltd. (Sichuan, China) (Supplementary Figure S1). Triplicate samples were obtained from three different points near the pit center on days $0,7,14,20,30$, and 40 . For isolation of microorganisms, the samples were stored at $4^{\circ} \mathrm{C}$. For physiochemical properties analysis, the samples were stored at $-20^{\circ} \mathrm{C}$. For DNA extraction, the samples were pulverized homogeneously in liquid nitrogen and stored at $-80^{\circ} \mathrm{C}$.

\section{Measurement of Physiochemical Properties}

Two grams of fermented grains were suspended in $20 \mathrm{ml}$ sterile distilled water, soaked by rotational shaking at $100 \mathrm{rpm}$ for $3 \mathrm{~h}$ at room temperature and then centrifuged at $10,000 \times g$ for $15 \mathrm{~min}$. The supernatant extract was collected for butyric acid and lactic acid analysis, which were determined by high performance liquid chromatography (HPLC) method as follows. The clear supernatant $(5 \mathrm{ml})$ was mixed thoroughly with $2 \mathrm{ml}$ zinc sulfate $(300 \mathrm{~g} / \mathrm{L})$ and $2 \mathrm{ml}$ potassium ferrocyanide $(106 \mathrm{~g} / \mathrm{L})$. The mixture was centrifuged at $10,000 \times \mathrm{g}$ for $10 \mathrm{~min}$, followed by filtration through a $0.22 \mu \mathrm{m}$ nylon syringe filter (ALWSCI, Zhejiang, China). A Waters E2695 HPLC system with a 2998 PDA detector was used for two kinds of acids assessment. Determination of lactic acid was performed using a Waters Atlantis T3 column $(5 \mu \mathrm{m}, 4.6 \mathrm{~mm} \times 250 \mathrm{~mm})$ with $30^{\circ} \mathrm{C}$ column temperature and $20 \mathrm{mM} \mathrm{NaH}_{2} \mathrm{PO}_{4}$ as the mobile phase ( $\mathrm{pH} 2.7,0.7 \mathrm{ml} / \mathrm{min}$ ). As for butyric acid analysis, the SEPAX Carbomix H-NP 5:8 column $(5 \mu \mathrm{m}, 7.8 \mathrm{~mm} \times 300 \mathrm{~mm})$ temperature was maintained at $55^{\circ} \mathrm{C}$ and the flow of mobile phase $\left(2.5 \mathrm{mM} \mathrm{H}_{2} \mathrm{SO}_{4}\right)$ was $0.6 \mathrm{ml} / \mathrm{min}$. For both of them, the injection amount was $10 \mu \mathrm{l}$ and detection wavelength was $210 \mathrm{~nm}$.

Temperature during fermentation process was measured with electronic temperature probes (PXTONG, PX-12). Ethanol content of fermented grains was detected according to the protocol described by Shen (2011). pH was detected using a Mettler Toledo FiveEasy Plus ${ }^{\mathrm{TM}} \mathrm{pH} / \mathrm{mV}$ meter equipped with an LE438 solid electrode (Mettler Toledo Instruments, Shanghai, China). Moisture content of fermented grains was determined immediately after sampling through a gravimetric approach by drying at $105^{\circ} \mathrm{C}$ for $4 \mathrm{~h}$ in the oven. All experiments were performed in three replicates.

\section{DNA Extraction and PCR Amplification}

DNA was extracted from fermented grains using the PowerSoil ${ }^{\circledR}$ DNA Isolation Kit (MOBIO Laboratories, Inc., Carlsbad, CA, USA) following the manufacturer's protocol. For each reaction, $0.5 \mathrm{~g}$ pulverized powder was used. The quality and quantity of DNA extracted was measured using a NanoDrop 3300 spectrophotometer (Wilmington, USA).

All primers used in this study are shown in Supplementary Table S1. Degenerate primers for buk and but genes were designed via the CODEHOP method ${ }^{1}$ (Rose et al., 2003; Chai et al., 2019). The but and buk genes were amplified as described by Vital et al. (2013) with some modifications. The PCR programs consisted of an initial 5 min denaturation at $94^{\circ} \mathrm{C}$, followed by 35 cycles of denaturation at $94^{\circ} \mathrm{C}$ for $30 \mathrm{~s}$, annealing at $47^{\circ} \mathrm{C}($ buk $)$ or $49^{\circ} \mathrm{C}$ (but) for $30 \mathrm{~s}$, extension at $72^{\circ} \mathrm{C}$ for $30 \mathrm{~s}$, and a final elongation step of $5 \mathrm{~min}$ at $72^{\circ} \mathrm{C}$.

With regard to bacterial consortia analysis in fermented grains, the V1/V3 hypervariable region (Escherichia coli positions 5-534) of 16S rRNA gene was amplified with the P1/P2 primer set (Huang et al., 2011). The Clostridia-specific primer set SJ-F/SJ-R targeting V4/V5 hypervariable region (E. coli positions 679-952) of 16S rRNA gene was used to specifically unravel the Clostridial community in fermented grains (Hu et al., 2014). The detailed PCR programs of these two primer sets were described in previous reports. At the $5^{\prime}$ end of each primer set used for high-throughput sequencing, unique multinucleotide sequences (18 bases) were synthesized as barcodes to assign sequences to different samples. The bacterial 27F/1492R primer set was used to amplify nearly full length $16 \mathrm{~S}$ rRNA gene of the isolated strains (Zhang et al., 2014), and the PCR program was as follows: one cycle at $95^{\circ} \mathrm{C}$ for $5 \mathrm{~min}$, followed by 30 cycles at $95^{\circ} \mathrm{C}$ for $60 \mathrm{~s}, 55^{\circ} \mathrm{C}$ for $30 \mathrm{~s}$ and $72^{\circ} \mathrm{C}$ for $90 \mathrm{~s}$, and a final extension at $72^{\circ} \mathrm{C}$ for $5 \mathrm{~min}$.

\section{Processing and Analyzing Bacterial 16S rRNA Gene Amplicon Sequencing Data}

To characterize the bacterial community in fermented grains, equivalent concentration of the barcoded 16S rRNA gene amplicons targeting V1/V3 hypervariable region were pooled and sequenced using a Roche/454 Genome Sequencer FLX Titanium. Mean length of the produced reads was $400 \mathrm{bp}$. The sequencing data was processed and analyzed via MOTHUR (version 1.35.1) according to the previous report (Xiao et al., 2017). To generate clean reads, reads shorter than 150 bp were discarded and reads with average quality score $<20$ or unknown nucleotides or any homopolymers of more than eight bases or without the primer sequence were removed from the raw data. Chimeric sequences identified by UCHIME were discarded as well (Edgar et al., 2011). Afterward, high-quality reads were clustered into individual OTUs at a $97 \%$ identity threshold

'https://4virology.net/virology-ca-tools/j-codehop/ 
via UPARSE$^{2}$ (Edgar, 2013). Afterward, the representative sequence from each OTU was aligned against the $\mathrm{RDP}^{3}$ and Greengenes (version gg_13_5) databases at a minimum of $80 \%$ identity for taxonomic annotation. OTUs that were not classified to the genus level were aligned to the EzBioCloud database (Yoon et al., 2017). The basic sequencing information and a-diversity indices were summarized in Supplementary Table S2.

The KEGG Ortholog (KO) functional profiles of microbial communities were predicted based on 16S rRNA gene sequences via the PICRUSt approach (Phylogenetic Investigation of Communities by Reconstruction of Unobserved States), which was performed following the workflow in Galaxy web ${ }^{4}$ under default parameter values (Langille et al., 2013). Referring to the butanoate metabolism pathway (ko00650), taxonomic distributions of potential butyrate-producing microbes in fermented grains were analyzed depending on buk (butyrate kinase, EC 2.7.2.7) and ptb (phosphate butyryltransferase, EC 2.3.1.19) in buk pathway; and atoD (acetate CoA/acetoacetate CoA-transferase alpha subunit, EC 2.8.3.8 2.8.3.9), atoA (acetate CoA/acetoacetate CoA-transferase beta subunit, EC 2.8.3.8 2.8.3.9) and ACSM (medium-chain acyl-CoA synthetase, EC 6.2.1.2) in but pathway. Then, Spearman's rank correlations between the predicted butyrate producers' succession and physiochemical properties dynamics during the fermentation process of fermented grains were assessed by SPSS (version 20.0) based on the squared Euclidean distance. Correlation coefficient $r \geq 0.8$ or $\leq-0.8$ and $p<0.05$ were set as cutoffs to evaluate the probability and significance.

\section{Clone Library Analysis of but and buk Partial Gene Amplicons}

Clone libraries based on buk and but partial gene amplicons were constructed to investigate the microorganisms with butyrate-producing potential. The PCR reaction was performed with ExTaq DNA polymerase (TAKARA, Dalian, China) with a total volume of $25 \mu \mathrm{l}$ and $20 \mathrm{ng}$ DNA from a mixture of each sample as template. The target PCR products located around $500 \mathrm{bp}(b u k)$ and $450 \mathrm{bp}$ (but) were purified using GK 2043-200 gel extraction kit (GENERAY, Shanghai, China). Then enriched amplicons were ligated with pMD19-T vector (TAKARA, Dalian, China) and transformed into E. coli JM109 competent cells. At least 100 recombinant clones were randomly picked for each gene and sent to Sangon Biotech (Shanghai, China) for sequencing. Nucleotide sequences were firstly trimmed in order to avoid the influences of degenerate bases and then clustered into operational taxonomic units (OTUs) under the threshold of $95 \%$ identity by Fungene Pipeline ${ }^{5}$. The OTUs were aligned against the UniProtKB database ${ }^{6}$ through BLASTx to determine their functional annotation and taxonomic classification. Their phylogenetic analysis based

${ }^{2}$ http://www.drive5.com/uparse/

${ }^{3}$ http://rdp.cme.msu.edu/classifier/classifier.jsp

${ }^{4}$ http://huttenhower.sph.harvard.edu/galaxy

${ }^{5}$ http://fungene.cme.msu.edu/FunGenePipeline/

${ }^{6} \mathrm{http}: / /$ www.uniprot.org/blast/ on neighbor-joining algorithm was conducted and visualized using MEGA version 7 (Kumar et al., 2016).

\section{Processing and Analyzing Clostridial- Specific 16S rRNA Gene Amplicon Sequencing Data}

Amplicons of Clostridia-specific V4/V5 hypervariable region of 16S rRNA gene were purified using a SanPrep Column PCR Product Purification Kit (Sangon Biotech, Shanghai, China). Barcoded amplicons were pooled in equal concentrations and sequenced using Illumina MiSeq Benchtop Sequencer $(2 \times 300$ bp). Quality-filtration and analysis of the sequencing data was performed following the standard workflow in QIIME (version 1.9.1) (Caporaso et al., 2010; Bokulich et al., 2013). The raw datasets were initially demultiplexed based on barcode and primer prior to assembly using FLASH (version 1.2.7) (Magoč and Salzberg, 2011). After quality filtration under QIIME pipeline and chimera removal using the UCHIME algorithm (Edgar et al., 2011), clean tags were clustered into OTUs at $97 \%$ similarity by UPARSE (Edgar, 2013). The representative sequences were then assigned to taxonomy with the Greengenes (version gg_13_5) and RDP classifier using $80 \%$ identity as a cutoff on QIIME. OTUs that were not classified to the genus level were aligned to the EzBioCloud database (Yoon et al., 2017). The basic sequencing information and $\alpha$-diversity indices were summarized in Supplementary Table S2. The phylogenetic trees displaying the dynamic changes of Clostridial community in fermented grains during brewing were constructed using MEGAN 5.10.6 (Mitra et al., 2011). Functional composition of Clostridia in butyrate metabolism pathway during the fermentation process was predicted by PICRUSt (Langille et al., 2013).

\section{S rRNA Gene-Guided Isolation of Clostridium and Metabolite Analysis by HPLC}

Based on the OTUs' sequences from Clostridia-specific 16S rRNA gene sequencing, species-specific culture media of Clostridium were predicted by a phylogeny-based predictor, called GROWREC, in the Known Media Database (KOMODO, http://komodo.modelseed.org) under default parameters (Oberhardt et al., 2015). The Clostridium strains were isolated by the predicted media from the fermented grains under an anaerobic system (DG250, Don Whitely Scientific, UK), including $80 \% \mathrm{~N}_{2}, 10 \% \mathrm{CO}_{2}$, and $10 \% \mathrm{H}_{2}$. DNA of the isolated strains was extracted by a Bacterial Genomic DNA Extraction Kit (DP302) (Tiangen, Beijing, China). Their 16S rRNA genes were amplified with $27 \mathrm{~F} / 1492 \mathrm{R}$ primer set (Zhang et al., 2014) and the obtained sequences were blasted against EzBioCloud database for taxonomic verification (Yoon et al., 2017). The potential butyrate-producing pathway for each verified strain was detected via but and buk genes amplification with but-F/but-R and buk-F/buk-R primer sets, respectively.

To quantify short-chain fatty acids (SCFA), the isolates were cultured in liquid reinforced clostridial medium (RCM, $\mathrm{pH}$ 6.5) at $37^{\circ} \mathrm{C}$ for 7 days in the above-mentioned anaerobic system. 
RCM included $10.0 \mathrm{~g} / \mathrm{L}$ peptone, $10.0 \mathrm{~g} / \mathrm{L}$ beef extract, $3.0 \mathrm{~g} / \mathrm{L}$ yeast extract, $5.0 \mathrm{~g} / \mathrm{L}$ glucose, $1.0 \mathrm{~g} / \mathrm{L}$ soluble starch, $0.5 \mathrm{~g} / \mathrm{L}$ cysteine hydrochloride, $3.0 \mathrm{~g} / \mathrm{L}$ sodium chloride, and $3.0 \mathrm{~g} / \mathrm{L}$ sodium acetate with $1.0 \mathrm{mg} / \mathrm{L}$ resazurin (a redox reaction indicator). Production of SCFA for each isolate was analyzed using the same HPLC workflow as butyrate described in section "Measurement of Physiochemical Properties." All culture experiments were performed in three biological replicates. The $16 \mathrm{~S}$ rRNA gene sequences of the isolates have been deposited in NCBI/GenBank database with the accession numbers MK720976-MK720991.

\section{RESULTS}

\section{Physiochemical Features of Fermented Grains Throughout Fermentation}

Variability patterns of fermented grains' physiochemical properties during brewing were recorded (Supplementary Figure S2). Temperature increased by around $10^{\circ} \mathrm{C}$ at the first 7 days of fermentation, afterward, decreased from 33 to $25^{\circ} \mathrm{C}$ till the end, changing about $2^{\circ} \mathrm{C}$ per 10 days. The accumulation of ethanol mainly occurred along with the temperature upregulation. Water content of fermented grains fluctuated between 53 and 57\% during fermentation. Acid fermentation environment was observed by $\mathrm{pH}$ detection. Lactic acid was one of the major organic acids in fermented grains, which had the tendency of upregulation through fermentation. As for butyric acid content, dramatical increase happened primarily from day 0 to 14 , and kept relatively stable until the end of fermentation (Figure 1B).

\section{Predictive Distribution of Key Enzymes Involved in Bacterial Butyrate Metabolism Pathways}

With respect to bacterial butyrate metabolism, enzymes ptb and buk in buk pathway and atoD, atoA, and ACSM in but pathway was considered to be directly related to the conversion between butyryl-CoA and butyrate (Figure 1A). It should be mentioned that but is named acetate CoA/acetoacetate CoA-transferase (i.e., atoD and atoA) in the KEGG Orthology system. Thus, temporal taxonomic distribution of these crucial enzymes during brewing was assessed based on 16S rRNA gene sequencing data and their metabolic profiles inferring by PICRUSt analysis. Qualified resulting reads from the amplicon sequencing of $16 \mathrm{~S}$ rRNA gene's V1-V3 hypervariable region were classified into 942 OTUs. Predictive functional profiling of microbial communities demonstrated buk pathway was the potential major butyrate biosynthesis pathway (Figure 2A). Bacilli, Bacteroidia, and Clostridia were identified as putative dominant bacteria involved in butyrate synthesis in fermented grains throughout fermentation of Luzhou-flavor baijiu; nevertheless, these three classes displayed divergent butyrate metabolic features.

OTUs classified as members of the Bacilli class were found to be the most abundant in fermented grains during brewing with relative abundance varying from 63.83 to $97.44 \%$ (Figure 2B). Bacilli was considered as the major potential butyrate-producing community in the fermented grains at the very beginning of fermentation (day 0). Bacillaceae and Staphylococcaceae families dominated Bacilli on day 0 (Figure 2C), and along with fermentation, their relative abundance dropped dramatically within a week, while Lactobacillaceae became prevailing till the end of fermentation (Supplementary Figure S3). Nevertheless, since the beginning of fermentation, the read numbers of $\mathrm{ptb}$ and buk significantly decreased, indicating Bacilli was no longer the major source of the butyrate producing key enzymes. It should be mentioned that a significant correlation $(r=0.812, p=0.001)$ was found between dynamics of lactate, the major organic acid in fermented grains, and the succession of Bacilli in fermented grains. Moreover, among the three predominant classes in fermented grains, the correlation relationship between butyrate and Clostridia ( $r=0.835, p=0.019)$ was the closest, followed by Bacilli $(r=0.670$, $p=0.012)$, while no significant correlation was identified for Bacteroidia $(r=0.099, p=0.748)$. Since there is no record of real but from Bacilli in FunGene database ${ }^{7}$, the predictive atoD and atoA in Bacilli might represent other kinds of CoA transferases.

Compared with Bacilli, Clostridia (mean relative abundance $=$ $2.21 \%$ ), and Bacteroidia (mean relative abundance $=2.93 \%$ ) were observed with rather exiguous abundance during the fermentation process (Figure 2B). Both the buk and but pathways were detected in Clostridia, while only the buk pathway was identified in Bacteroidia (Figure 2A). Presumptive Bacteroidia butyrate producers could only be detected in the fermented grains on days 7, 14, 20, and 30, and Prevotellaceae (order Bacteroidales) was the predominant butyrate-producing Bacteroidia community in the sample on day 7 (Figure 2D). Clostridia was identified as the potential butyrate producers throughout fermentation and dominated butyrate production in the samples on days 14,20 , 30, and 40 (Figure 2A). Furthermore, the hiatus of ACSM in all samples suggested that butyrate in fermented grains was not widely utilized as a carbon source by bacteria.

\section{Clone Library Analysis of buk and but Genes}

In order to evaluate the predictive functional profiles of bacterial community and further investigate butyrate-producing bacteria in fermented grains, we performed clone library analysis based on the key-enzyme coding genes buk and but in butyrate synthesis pathway. A total of 100 clones from each clone library were assigned to 11 OTUs and seven OTUs, respectively (Figure 3).

As for the buk clone library, all the OTUs were confirmed as real buk sequences (Figure 3A). According to the BLASTx results, OTU2 (relative abundance $=14 \%$ ), OTU8 $(6 \%)$, and OTU11 (1\%) fell into class Bacilli; OTU7 (6\%) belonged to Bacteroidia; relative abundance of the left seven OTUs accounted for $73 \%$ of all identified buk clones that were scattered in Clostridia and five of them were further annotated as different species of genus Clostridium (Supplementary Table S3). With

${ }^{7}$ http://fungene.cme.msu.edu/index.spr 

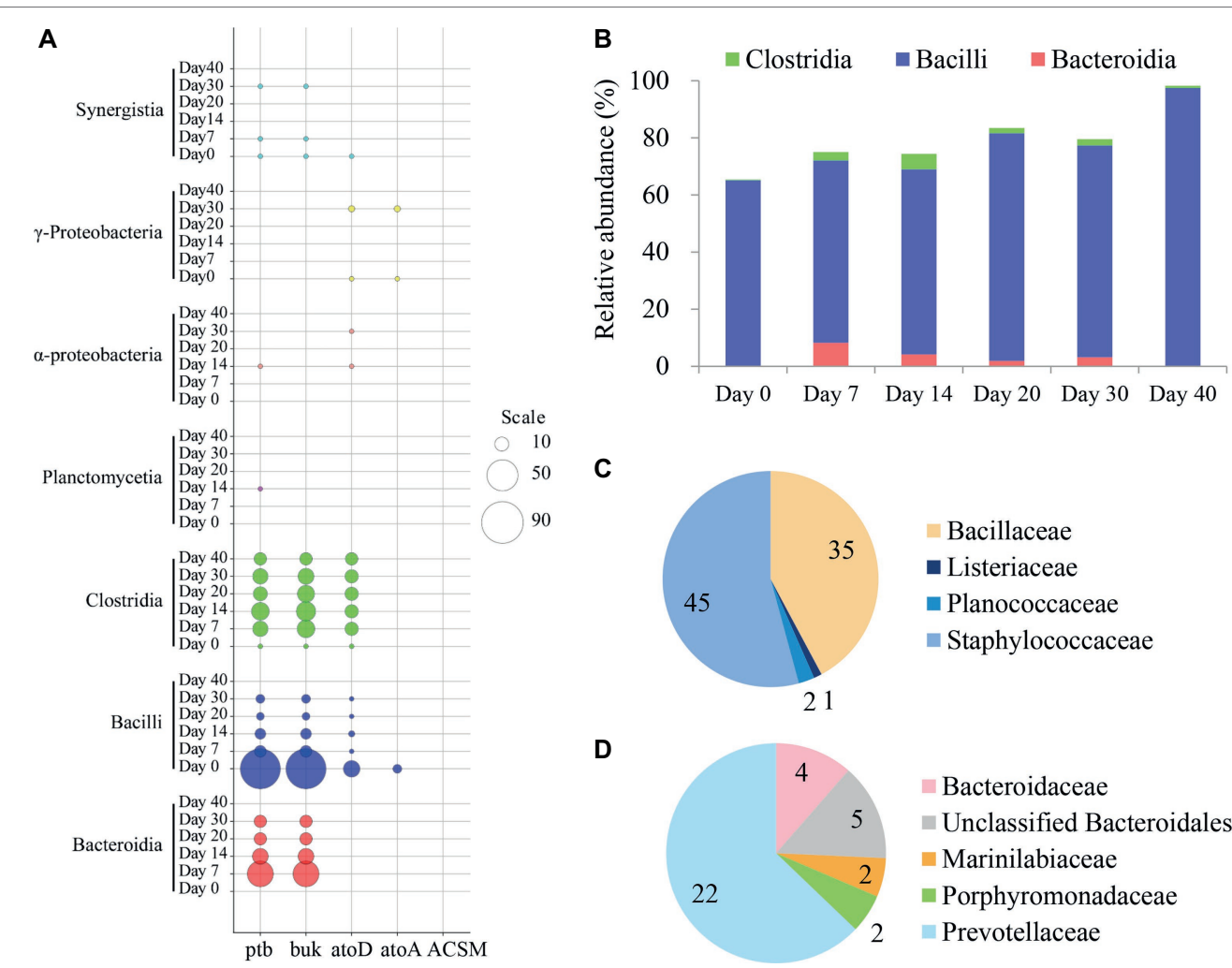

C

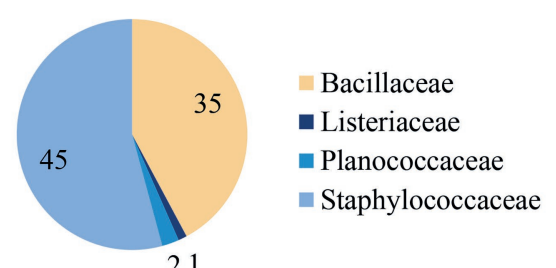

D

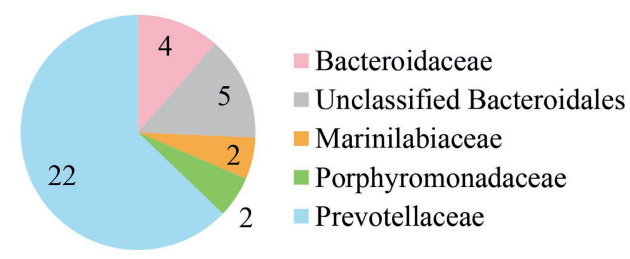

FIGURE 2 | Temporal distribution of butyrate producers in the fermented grains of Luzhou-flavor baijiu. (A) Taxonomic distribution and enzymes (ptb, buk, atoD, atoA and ACSM) reads for butyrate formation in the microbial community of fermented grains via PICRUSt analysis. The diameters of bubbles (scale) are proportional to the read numbers of enzymes. (B) Succession of three major classes (Clostridia, Bacilli, and Bacteroidia) related to butyrate synthesis based on sequencing of $16 \mathrm{~S}$ rRNA gene. (C) Family-level diversity of buk-associated Bacilli in the fermented grains on day 0 . The numbers represent the read numbers of buk. (D) Family-level diversity of buk-associated Bacteroidia in the fermented grains on day 7 . The numbers represent the read numbers of buk.

regard to the but gene (Figure $3 \mathbf{B}$ ), due to its high sequence similarity to 4-hydroxybutyrate CoA-transferase (4-Hbt) (Charrier et al., 2006), which also participated in the conversion of butyryl-CoA to butyrate (Louis and Flint, 2017), we failed to exclude the amplification of these distinct genes. Only OTU3 (12\%), OTU6 (3\%), and OTU7 (2\%) were confirmed as real but sequences. Since the subtle similarities to both but and 4-Hbt gene were detected, OTU1 (56\%) and OTU4 (5\%) were also considered as candidate but sequences (Supplementary Table S4). The closest reference sequences of all but OTUs belonged to Clostridia. Based on this part, Clostridia was considered as the potential major butyrate-producing bacteria in both buk and but pathways. Comprehensive considerations combined with functional prediction, we decided to investigate the Clostridial community via Clostridia-specific 16S rRNA gene sequencing analysis to further zoom in on the Clostridia with butyrate-producing potential in fermented grains.

\section{Taxonomic Diversity of Clostridia and their Butyrate Metabolic Profiling Prediction}

As Clostridia was not predominant in microbial community of fermented grains (Figure 2B), using universal bacterial primer sets for sequencing analysis possibly caused the loss of information about functioning Clostridia irrespective of their abundance. Therefore, Clostridial specific primer set SJ-F/SJ-R was applied for sequencing to interpret the taxonomic diversity of Clostridia. After quality-filtration, 20,000 reads per sample were clustered into 2,779 OTUs in total based on $3 \%$ dissimilarity of $16 \mathrm{~S}$ rRNA gene sequences. Among them, 89.43\% OTUs were affiliated with Clostridia, while the rest were assigned to class Negativicutes (further annotated as family Veillonellaceae), which formerly belonged to Clostridial cluster IX (Marchandin et al., 2010). At the order level, Clostridia was dominated by Clostridiales (98.72\%), of which $29.23 \%$ remained unclassified at the family level. As for the taxonomic distribution at the genus level, 152 OTUs with the average relative abundance per sample $>0.1 \%$ were selected for analysis. A total of 18 genera scattered in 10 families (Clostridiaceae, Clostridiales Incertae Sedis Family XI and Family XIII, Lachnospiraceae, Eubacteriaceae, Gracilibacteraceae, Peptococcaceae, Peptostreptococcaceae, Ruminococcaceae, and Syntrophomonadaceae) were identified in fermented grains throughout fermentation (Figure 4, Supplementary Figure S4). The relative abundance of Clostridium was the highest among the 18 identified genera, followed by Sedimentibacter. Subsequently, their potential metabolic features 

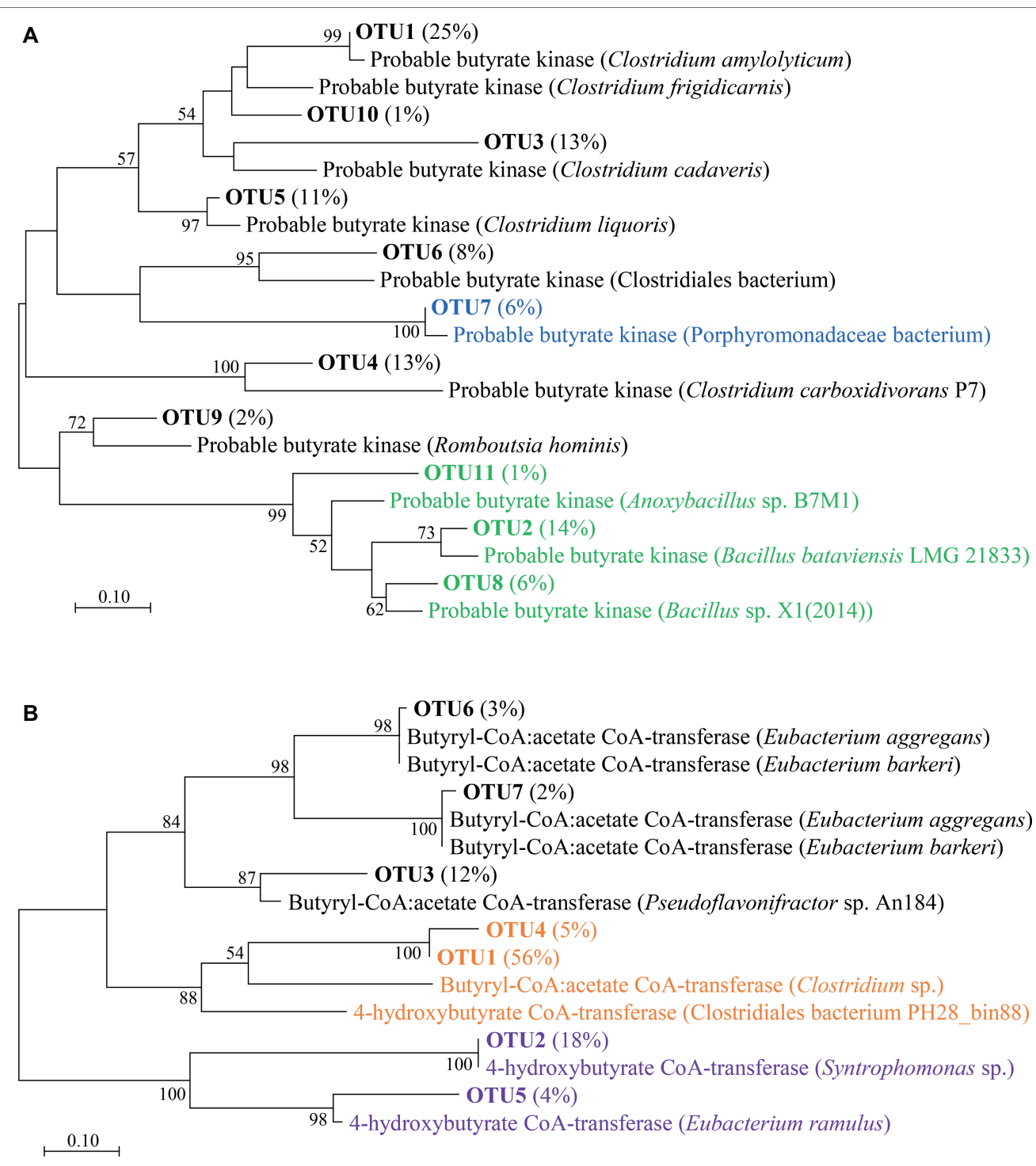

FIGURE 3 | Phylogenetic diversities of buk and but genes in the fermented grains of Luzhou-flavor baijiu. (A) A neighbor-joining tree of deduced protein sequences of buk genes. Green, blue and black branches indicate Bacilli, Bacteroidia and Clostridia, respectively. (B) A neighbor-joining tree of deduced protein sequences of but genes. Black, orange and purple branches indicate real but, candidate but and 4-hydroxybutyrate CoA-transferase gene, respectively. For (A) and (B), bootstrap values from 1,000 replicates are included. Percentage in the bracket shows the relative abundance of specific OTU.

were analyzed via PICRUSt to further reveal Clostridial butyrate producers.

The family-level distribution of potential Clostridia butyrate producers is displayed in Figure 5. In agreement with the PICRUSt analysis results of bacterial community, here buk pathway was also considered as the possibly major synthetic pathway of butyrate in fermented grains. Clostridiaceae (mean relative abundance $=38.12 \%$ ) and [Tissierellaceae] (mean relative abundance $=12.30 \%$ ) were the predominant contributors of butyrate synthesis key enzymes, including ptb, buk, and atoD. Family [Tissierellaceae] was classified in the Clostridiales Incertae Sedis Family XI with the RDP database. The buk pathway was mainly observed in Clostridiaceae, [Tissierellaceae],
Christensenellaceae, Lachnospiraceae, and [Mogibacteriaceae], while the but pathway was merely identified in Clostridiaceae, [Tissierellaceae], and [Mogibacteriaceae]. Read numbers of these enzymes demonstrated different variation patterns along with fermentation. In general, read numbers of ptb, buk, and atoD in [Tissierellaceae] and atoD in Clostridiaceae were downregulated, while upregulation of ptb and buk was detected in Christensenellaceae (Figure 5). It is worth noting that read numbers of ptb and buk in Clostridiaceae almost remained constant during brewing, and ranked first compared with other families. As Clostridium dominated Clostridiaceae (Figure 4), we deduced that Clostridium might be the crucial butyrate producers in fermented grains based on the above-mentioned studies. 


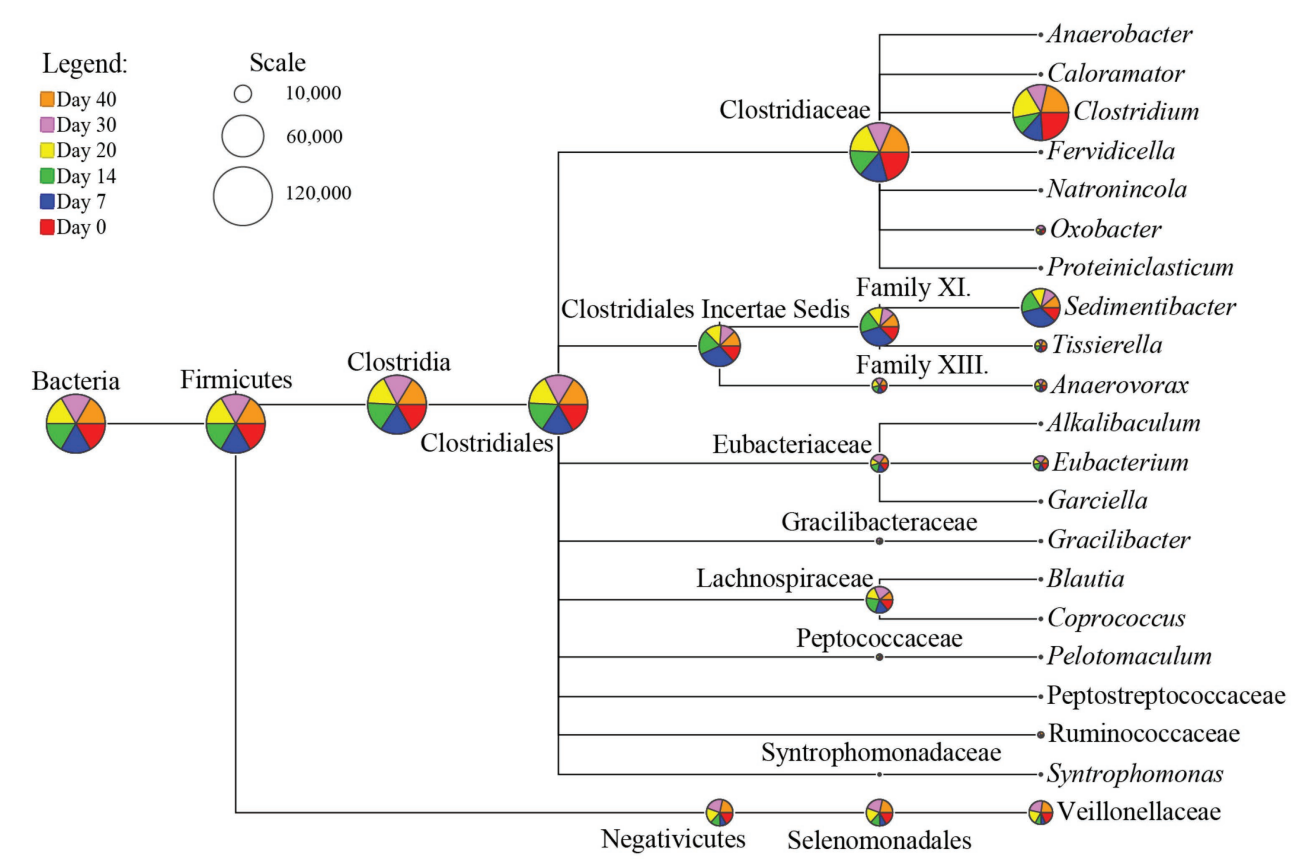

FIGURE 4 | Dynamic patterns of Clostridial consortia in the fermented grains during baijiu fermentation. The results were based on Clostridia-specific 16S rRNA gene sequencing analysis. Scale represents read numbers. Area of each pie chart in the node of phylogenetic tree is proportional to the square root of reads assigned.

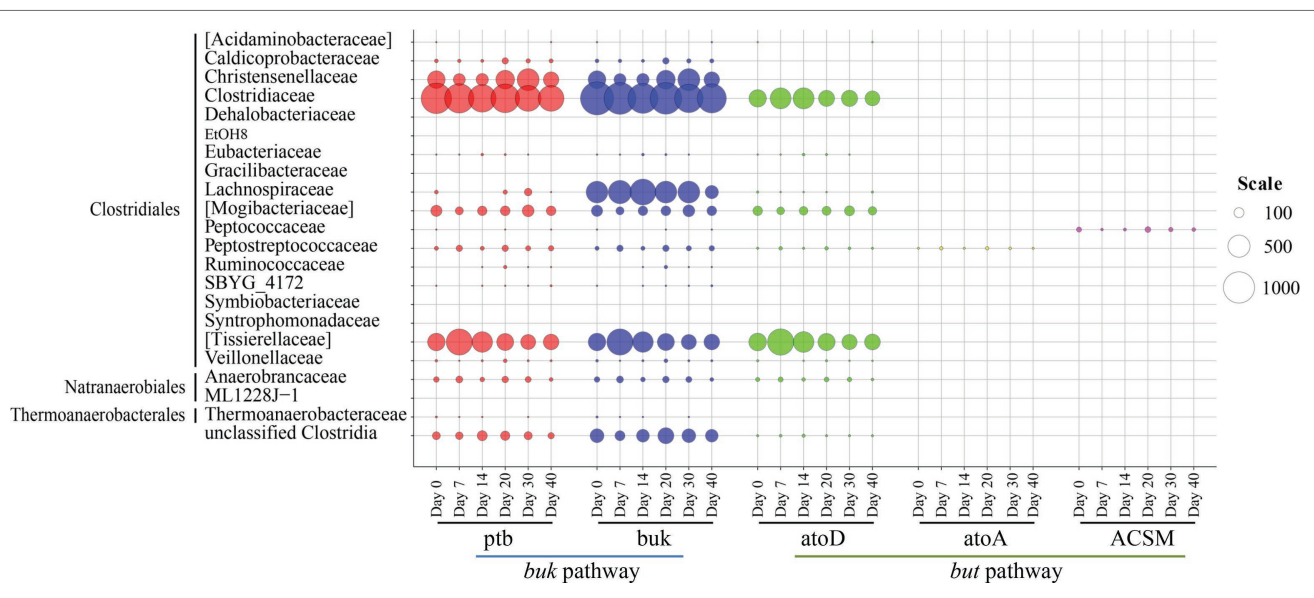

FIGURE 5 | Family-level distribution of Clostridia in butyrate synthetic pathways during baijiu fermentation analyzed by Clostridia-specific 16S rRNA gene sequencing and PICRUSt analysis. The diameter of bubbles (scale) is proportional to the read numbers of enzymes.

\section{Evaluation of Clostridium Butyrate- Producing Potential by Culture Method}

According to the Clostridia-specific 16S rRNA gene sequences, the media were predicted for Clostridia by GROWREC in KOMODO database. In total, 16 kinds of media for Clostridium (media ID in DSMZ database: 1, 58, 78, 110, 104b, 136, 144, 156, 453, 514, 671, 830, 872, 898.3, 915.2, and 960.4; link: http://komodo.modelseed.org/servlet/KomodoTomcatServerSide UtilitiesModelSeed?MediaList) were predicted based on the $16 \mathrm{~S}$
rRNA gene sequences of 42 OTUs belonging to genus Clostridium. A total of 17 Clostridium species were isolated from the fermented grains of Luzhou-flavor baijiu using the predicted media (Table 1).

After specific isolation, all Clostridium strains were cultured in the Clostridial universal medium RCM to assess and compare their butyrate-producing capacities. Among the 17 species of Clostridium, 12 species (C. aminovalericum, C. amylolyticum, C. butyricum, C. kluyveri, C. kogasensis, C. luticellarii, C. mangenotii, 
TABLE 1 | Profiles of short-chain fatty acids in the fermentation broth of Clostridium strains isolated with the media predicted by GROWREC in Known Media Database.

\begin{tabular}{|c|c|c|c|c|c|c|c|c|c|}
\hline \multirow{2}{*}{ Strains } & \multirow{2}{*}{$\begin{array}{c}\text { GenBank } \\
\text { accession no. }\end{array}$} & \multirow{2}{*}{$\begin{array}{l}\text { Identity } \\
(\%)\end{array}$} & \multirow{2}{*}{$\begin{array}{c}\text { Media ID in } \\
\text { the DSMZ } \\
\text { database }^{\text {b }}\end{array}$} & \multicolumn{2}{|c|}{$\begin{array}{c}\text { Butyrate synthesis } \\
\text { pathways }\end{array}$} & \multicolumn{4}{|c|}{ Production of fatty acids $(g / L)^{c}$} \\
\hline & & & & buk & but & Acetate & Butyrate & Pentanoate & Caproate \\
\hline $\begin{array}{l}\text { Clostridium } \\
\text { acetobutylicum }\end{array}$ & KJ951058.1 & 100 & 156 & $--^{a}$ & - & $0.23 \pm 0.00$ & $N^{d}$ & ND & ND \\
\hline $\begin{array}{l}\text { Clostridium } \\
\text { aminovalericum }\end{array}$ & NR_113199.1 & 99 & $104 b$ & + & - & $1.28 \pm 0.11$ & $6.70 \pm 0.47$ & $1.43 \pm 0.06$ & $3.20 \pm 0.21$ \\
\hline $\begin{array}{l}\text { Clostridium } \\
\text { amylolyticum }\end{array}$ & NR_044386.1 & 99 & 136 & + & - & $0.18 \pm 0.47$ & $7.71 \pm 0.52$ & ND & $0.33 \pm 0.05$ \\
\hline $\begin{array}{l}\text { Clostridium } \\
\text { botulinum }\end{array}$ & CP013243.1 & 100 & $104 b$ & + & - & $0.93 \pm 0.06$ & ND & $1.89 \pm 0.08$ & $2.63 \pm 0.13$ \\
\hline $\begin{array}{l}\text { Clostridium } \\
\text { butyricum }\end{array}$ & HQ328064 & 100 & 1 & + & - & $1.22 \pm 0.13$ & $1.52 \pm 0.05$ & $0.03 \pm 0.01$ & ND \\
\hline $\begin{array}{l}\text { Clostridium } \\
\text { carboxidivorans }\end{array}$ & CP011803.1 & 99 & 156 & + & - & $1.49 \pm 0.08$ & ND & $0.36 \pm 0.08$ & $0.49 \pm 0.07$ \\
\hline $\begin{array}{l}\text { Clostridium } \\
\text { kluyveri }\end{array}$ & NR_074447.1 & 100 & 220 & - & + & ND & $0.34 \pm 0.05$ & ND & $1.65 \pm 0.23$ \\
\hline $\begin{array}{l}\text { Clostridium } \\
\text { kogasensis }\end{array}$ & NR_136452.1 & 99 & 110 & + & + & $1.82 \pm 0.08$ & $0.54 \pm 0.02$ & $1.34 \pm 0.13$ & $0.79 \pm 0.11$ \\
\hline $\begin{array}{l}\text { Clostridium } \\
\text { luticellarii }\end{array}$ & KP342257.1 & 99 & 220 & - & + & ND & $0.13 \pm 0.02$ & ND & ND \\
\hline $\begin{array}{l}\text { Clostridium } \\
\text { mangenotii }\end{array}$ & NR_104729.1 & 100 & 1 & + & - & ND & $0.09 \pm 0.01$ & ND & ND \\
\hline Clostridium novyi & LC193834.1 & 99 & 156 & - & + & $0.70 \pm 0.66$ & $5.77 \pm 0.39$ & $1.93 \pm 0.09$ & $2.80 \pm 0.11$ \\
\hline $\begin{array}{l}\text { Clostridium } \\
\text { senegalense }\end{array}$ & NR_125591.1 & 100 & 58 & - & - & $1.07 \pm 0.02$ & ND & $2.17 \pm 0.10$ & $3.03 \pm 0.18$ \\
\hline $\begin{array}{l}\text { Clostridium } \\
\text { sporogenes }\end{array}$ & NR_029231.1 & 100 & $104 b$ & + & - & $0.35 \pm 0.06$ & $0.28 \pm 0.04$ & ND & $3.20 \pm 0.21$ \\
\hline $\begin{array}{l}\text { Clostridium } \\
\text { swellfunianum }\end{array}$ & NR_126179.1 & 97 & $104 b$ & - & - & $0.88 \pm 0.05$ & ND & ND & ND \\
\hline $\begin{array}{l}\text { Clostridium } \\
\text { tyrobutyricum }\end{array}$ & СР014170.1 & 100 & $104 b$ & - & + & $1.05 \pm 0.08$ & $0.43 \pm 0.06$ & $0.64 \pm 0.03$ & ND \\
\hline $\begin{array}{l}\text { Clostridium sp. } \\
\text { ArC5 }\end{array}$ & AF443594.1 & 99 & $104 b$ & + & - & $0.85 \pm 0.00$ & $4.00 \pm 0.22$ & $2.13 \pm 0.11$ & $2.84 \pm 0.14$ \\
\hline $\begin{array}{l}\text { Clostridium sp. } \\
\text { YN5 }\end{array}$ & AB537983.1 & 97 & $104 b$ & + & - & ND & $0.25 \pm 0.03$ & ND & $1.53 \pm 0.11$ \\
\hline
\end{tabular}

Species highlighted by bold fonts were first isolated from baijiu brewing ecosystem according to Zou et al. (2018a) and Chai et al. (2019). aNo visible band around correct size. ${ }^{b}$ 1, nutrient agar; 58, Bifidobacterium medium; 104b, PYX medium; 110, chopped meat medium with carbohydrates; 136, Veillonella medium; 156, Clostridium propionicum medium; 220, CASO Agar (Merck 105458); 520, Clostridium cellulolyticum (CM3). According to the medium number, the detailed medium formulation can be found from the following link: http://komodo.modelseed.org/servlet/KomodoTomcatServerSideUtilitiesModelSeed?MediaList

'Values are expressed as means \pm standard deviation.

${ }^{d}$ Not detected or the peak area is over 100-fold smaller than internal standard.

C. novyi, C. sporogenes, C. tyrobutyricum, C. sp. ArC5, and C. sp. YN5) could produce butyrate, wherein the butyrate production of $C$. amylolyticum was the highest $(7.71 \mathrm{~g} / \mathrm{L})$, followed by C. aminovalericum $(6.70 \mathrm{~g} / \mathrm{L})$, C. novyi $(5.77 \mathrm{~g} / \mathrm{L})$, and C. sp. ArC5 $(4.00 \mathrm{~g} / \mathrm{L})$ (Table 1). Here, Clostridium amylolyticum (Song and Dong, 2008), C. mangenotii (Lawson et al., 2016), and C. sp. ArC5 were first identified with butyrateproducing potential, possibly due to different culture conditions. With regard to the butyrate synthesis genes, Clostridium aminovalericum, C. amylolyticum, C. butyricum, C. mangenotii, C. sporogenes, C. sp. ArC5, and C. sp. YN5 were found to have buk gene by PCR amplification, while but gene could be amplified in C. kluyveri, C. luticellarii, C. novyi, and C. tyrobutyricum. Clostridium kogasensis was the only one species possessing both buk and but genes.

\section{DISCUSSION}

After undergoing a multispecies solid-state fermentation process, Chinese baijiu is finally produced via distillation. Among the microbial metabolites, butyrate and its derivatives are important aroma compounds in Luzhou-flavor baijiu (Fan and Qian, 2006). Hence, studying the butyrate producers during the fermentation process could help to develop a deeper understanding of the fermentative mechanisms of Luzhouflavor baijiu and locate the key functional community contributing to the formation of its unique flavor. Though anaerobic bacteria, especially Clostridium, were considered as important butyrate producers in the pit mud of baijiu during the sealed-in-cellar manufacturing process (Hu et al., 2015; Liu et al., 2015), there was no report specifically elucidating 
butyrate-producing bacterial assembly in the fermentation grains of baijiu during brewing.

In this study, PICRUSt analysis based on the bacterial $16 \mathrm{~S}$ rRNA gene sequences revealed that the buk pathway could be the major butyrate biosynthesis pathway in fermented grains of Luzhou-flavor baijiu (Figure 2A). The buk gene was usually more abundant in the intestine of many carnivores, and the lack of consistent acetate supply was considered as the major factor that limit the performance of but (Vital et al., 2015). However, as the fermentation matrix, the fermented grains consisted entirely of sorghum and other grains; the relative high content of acetate in the fermented grains (around $1.40 \mathrm{~g} / \mathrm{kg}$ ) indicated that there might be other factors influencing the distribution of the two pathways in different environments.

At the class level, Bacilli, Clostridia, and Bacteroidia were found as three main potential butyrate-producing groups in fermented grains (Figure 2). Bacilli and Bacteroidia possessed the buk pathway, while Clostridia was able to produce butyrate via both the buk and but pathways. Bacillaceae and Staphylococcaceae belonging to Bacilli were the major potential contributors of butyrate kinase at the beginning of fermentation, which was also considered as associated with butyrate production in human gut (Magnúsdóttir et al., 2017). However, along with the fermentation, lactic acid bacteria, especially Lactobacillaceae became dominant, and possibly inhibited the proliferation of Bacillaceae and Staphylococcaceae by producing lactate. It should be mentioned that butyrate producers from Clostridia and Bacteroidia were not significantly affected by lactate, which related to their ability of utilizing lactate as a carbon source (Reichardt et al., 2014). Moreover, the spatial heterogeneity characterized the solidstate fermentation environment of baijiu, which could generate numerous ecological niches with divergent environmental factors. This was also conductive to explain the negligible impact of lactate on Clostridia and Bacteroidia. Prevotellaceae was the major Bacteroidia butyrate-producing group in fermented grains (especially on day 7). Nevertheless, Bacteroidia was found mainly composed by Bacteroides (Bacteroidaceae) and regarded as non-butyrate producer (Reichardt et al., 2014).

The buk and but pathways that directly involved in bacterial butyrate synthesis in fermented grains were further examined by clone library analysis of but and buk partial gene amplicons. The sequences of the buk gene mainly belonged to Clostridia, followed by Bacilli and Bacteroidia, while those of the but gene belonged to Clostridia (Figure 3). Due to the low identity to reference sequences $(<80 \%)$, some OTUs in these two libraries could not be annotated at the genus or species level. As the information in the reference database is improved, the taxonomic and functional assignment of sequencing reads in this study will be more accurate.

Similar to pit mud, Clostridia were also regarded as the major potential butyrate-producing bacteria in fermented grains (Chai et al., 2019). However, it was not widely studied in previous studies of fermented grains due to its low relative abundance as compared with lactic acid bacteria
(Zou et al., 2018b). Thus, diversity and succession of Clostridia in the fermented grains of Luzhou-flavor baijiu was systematically studied by Clostridia-specific $16 \mathrm{~S}$ rRNA gene sequencing. The majority of Clostridia belong to Clostridiales, in which Clostridiaceae and Clostridiales Incertae Sedis Family XI ([Tissierellaceae]) are the two dominant families. Clostridium (Clostridiaceae) was predicted as a major potential butyrate producer in the fermented grains (Figure 4). It stands to reason that endpoint metagenomes just tell us which organisms have been present at some point in the process but may have died and left their DNA in the actual sample. As such, unraveling the dynamic expression of genes in baijiu microbiota at transcriptional and protein level is ongoing in further studies, e.g., RNA-based DGGE and $16 \mathrm{~S}$ rRNA gene sequencing approaches have been used in elucidating functional microbes in food fermentation microbial ecosystem (Alessandria et al., 2016; Wuyts et al., 2018). Reinforced Clostridial Medium (RCM) and ethanol/sodium acetate (ES) medium were used to isolate various Clostridium strains from pit mud in the previous work (Chai et al., 2019). However, different from pit mud, Clostridium assembly was a tiny minority among bacteria in fermented grains, and merely four species of Clostridium were isolated from the fermented grains by RCM and ES media in our preliminary study (data not shown). To convince the results from molecular approach, a 16S rRNA gene sequence-guided culture medium prediction method was applied to isolate the Clostridium strains. A total of 16 media were successfully predicted by GROWREC in KOMODO database, which facilitated the isolation of strains belonging to 17 Clostridium species from the fermented grains (Table1). Five species (C. acetobutylicum, C. botulinum, C. kogasensis, C. mangenotii, and C. sp. YN5) have not been reported in the fermentation of baijiu in previous studies (Zou et al., 2018a; Chai et al., 2019). Among the 17 species, 12 species of Clostridium could produce butyrate in the fermentation broth via the buk and/or but pathways (Table 1), which validated the phylogenetic and functional diversities of butyrate producer in the fermented grains of baijiu. However, in pit mud, buk pathway might dominate the butyrate production of Clostridium via metagenomic analysis (Tao et al., 2017). The coupled metagenomics and GROWREC method might be used in future study for isolating other potential butyrate producers (even with low abundance) in the fermented grains, such as Sedimentibacter species.

In summary, this study revealed that Clostridia functioned as an important functional group driving the butyrate formation via the buk and but pathways by clone library analysis of but and buk partial gene amplicons, high throughput sequencing and PICRUSt functional analysis of $16 \mathrm{~S}$ rRNA genes. The genus Clostridium was found as the major potential butyrate producer among 18 genera in the class Clostridia by Clostridiaspecific 16S rRNA gene sequencing and PICRUSt analysis. A total of 17 species of Clostridium were successfully isolated by the GROWREC-aided method, and the profiles of fatty acids and functional genes (but and buk) in these species validated the phylogenetic and functional diversities of butyrateproducing bacteria in the baijiu microbiota. We plan to further 
elucidating the similarities and differences of bacterial butyratesynthesizing mechanisms between pit mud and fermented grains during brewing.

\section{DATA AVAILABILITY}

No datasets were generated or analyzed for this study.

\section{AUTHOR CONTRIBUTIONS}

Z-ML and Z-HX designed the experiments. JM, L-JC, P-XX, WQ, and CX conducted the experiments and analyzed the results. L-JC, Z-ML, and X-JZ prepared the manuscript. S-TW, $\mathrm{C}-\mathrm{HS}$, and J-SS assisted in drafting the manuscript. All authors read and approved the final manuscript.

\section{REFERENCES}

Alessandria, V., Ferrocino, I., De Filippis, F., Fontana, M., Rantsiou, K., Ercolini, D., et al. (2016). Microbiota of an Italian grana-like cheese during manufacture and ripening, unraveled by $16 \mathrm{~S}$ rRNA-based approaches. Appl. Environ. Microbiol. 82, 3988-3995. doi: 10.1128/AEM.00999-16

Bokulich, N. A., Subramanian, S., Faith, J. J., Gevers, D., Gordon, J. I., Knight, R., et al. (2013). Quality-filtering vastly improves diversity estimates from Illumina amplicon sequencing. Nat. Methods 10, 57-59. doi: 10.1038/nmeth.2276

Caporaso, J. G., Kuczynski, J., Stombaugh, J., Bittinger, K., Bushman, F. D., Costello, E. K., et al. (2010). QIIME allows analysis of high-throughput community sequencing data. Nat. Methods 7, 335-336. doi: 10.1038/nmeth.f.303

Chai, L. J., Xu, P. X., Qian, W., Zhang, X. J., Ma, J., Lu, Z. M., et al. (2019). Profiling the Clostridia with butyrate-producing potential in the mud of Chinese liquor fermentation cellar. Int. J. Food Microbiol. 297, 41-50. doi: 10.1016/j.ijfoodmicro.2019.02.023

Charrier, C., Duncan, G. J., Reid, M. D., Rucklidge, G. J., Henderson, D., Young, P., et al. (2006). A novel class of CoA-transferase involved in shortchain fatty acid metabolism in butyrate-producing human colonic bacteria. Microbiology 152, 179-185. doi: 10.1099/mic.0.28412-0

Ding, X., Wu, C., Huang, J., and Zhou, R. (2015). Interphase microbial community characteristics in the fermentation cellar of Chinese Luzhou-flavor liquor determined by PLFA and DGGE profiles. Food Res. Int. 72, 16-24. doi: 10.1016/j.foodres.2015.03.018

Edgar, R. C. (2013). UPARSE: highly accurate OTU sequences from microbial amplicon reads. Nat. Methods 10, 996-998. doi: 10.1038/nmeth.2604

Edgar, R. C., Haas, B. J., Clemente, J. C., Quince, C., and Knight, R. (2011). UCHIME improves sensitivity and speed of chimera detection. Bioinformatics 27, 2194-2200. doi: 10.1093/bioinformatics/btr381

Fan, W., and Qian, M. C. (2006). Identification of aroma compounds in Chinese "Yanghe Daqu" liquor by normal phase chromatography fractionation followed by gas chromatography/olfactometry. Flavour Fragr. J. 21, 333-342. doi: $10.1002 / \mathrm{ff} .1621$

Fang, C., Du, H., Jia, W., and Xu, Y. (2019). Compositional differences and similarities between typical Chinese baijiu and western liquor as revealed by mass spectrometry-based metabolomics. Meta 9:2. doi: 10.3390/metabo9010002

$\mathrm{Hu}, \mathrm{X}$. L., Du, H., and $\mathrm{Xu}, \mathrm{Y}$. (2015). Identification and quantification of the caproic acid-producing bacterium Clostridium kluyveri in the fermentation of pit mud used for Chinese strong-aroma type liquor production. Int. J. Food Microbiol. 214, 116-122. doi: 10.1016/j.ijfoodmicro.2015.07.032

Hu, X. L., Wang, H. Y., Wu, Q., and Xu, Y. (2014). Development, validation and application of specific primers for analyzing the clostridial diversity in dark fermentation pit mud by PCR-DGGE. Bioresour. Technol. 163, 40-47. doi: 10.1016/j.biortech.2014.04.008

\section{FUNDING}

This work was supported by the Key Program of National Natural Science Foundation of China (No. 31530055), National First-class Discipline Program of Light Industry Technology and Engineering (LITE2018-11), three grants from the National High-tech Research and Development Program (863 Project) (No. 2012AA021301, No. 2013AA102106, and No. 2014AA021501), and National Engineering Research Center of Solid-State Brewing (No. 38776).

\section{SUPPLEMENTARY MATERIAL}

The Supplementary Material for this article can be found online at: https://www.frontiersin.org/articles/10.3389/fmicb.2019.01397/ full\#supplementary-material

Huang, S., Yang, F., Zeng, X., Chen, J., Li, R., Wen, T., et al. (2011). Preliminary characterization of the oral microbiota of Chinese adults with and without gingivitis. BMC Oral Health 11:33. doi: 10.1186/1472-6831-11-33

Kumar, S., Stecher, G., and Tamura, K. (2016). MEGA7: molecular evolutionary genetics analysis version 7.0 for bigger datasets. Mol. Biol. Evol. 33, 1870-1874. doi: 10.1093/molbev/msw054

Langille, M., Zaneveld, J., Caporaso, J. G., McDonald, D., Knights, D., Reyes, J., et al. (2013). Predictive functional profiling of microbial communities using 16S rRNA marker gene sequences. Nat. Biotechnol. 31, 814-821. doi: 10.1038/nbt.2676

Lawson, P. A., Citron, D. M., Tyrrell, K. L., and Finegold, S. M. (2016). Reclassification of clostridium difficile as clostridioides difficile (Hall and O’Toole 1935) Prévot 1938. Anaerobe 40, 95-99. doi: 10.1016/j.anaerobe.2016.06.008

Liu, H., and Sun, B. (2018). Effect of fermentation processing on the flavor of Baijiu. J. Agric. Food Chem. 66, 5425-5432. doi: 10.1021/acs.jafc.8b00692

Liu, J., Wu, Q., Wang, P., Lin, J., Huang, L., and Xu, Y. (2017). Synergistic effect in core microbiota associated with sulfur metabolism in spontaneous Chinese liquor fermentation. Appl. Environ. Microbiol. 83, e01475-e01417. doi: 10.1128/AEM.01475-17

Liu, M., Zhao, K., Tang, Y., Ren, D., Yao, W., Tian, X., et al. (2015). Analysis of Clostridium cluster I community diversity in pit mud used in manufacture of Chinese Luzhou-flavor liquor. Food Sci. Biotechnol. 24, 995-1000. doi: 10.1007/s10068-015-0127-7

Louis, P., and Flint, H. J. (2009). Diversity, metabolism and microbial ecology of butyrate-producing bacteria from the human large intestine. FEMS Microbiol. Lett. 294, 1-8. doi: 10.1111/j.1574-6968.2009.01514.x

Louis, P., and Flint, H. J. (2017). Formation of propionate and butyrate by the human colonic microbiota. Environ. Microbiol. 19, 29-41. doi: $10.1111 / 1462-2920.13589$

Louis, P., Young, P., Holtrop, G., and Flint, H. J. (2010). Diversity of human colonic butyrate-producing bacteria revealed by analysis of the butyrylCoA:acetate CoA-transferase gene. Environ. Microbiol. 12, 304-314. doi: 10.1111/j.1462-2920.2009.02066.x

Magnúsdóttir, S., Heinken, A., Kutt, L., Ravcheev, D. A., Bauer, E., Noronha, A., et al. (2017). Generation of genome-scale metabolic reconstructions for 773 members of the human gut microbiota. Nat. Biotechnol. 35, 81-92. doi: $10.1038 /$ nbt.3703

Magoč, T., and Salzberg, S. L. (2011). FLASH: fast length adjustment of short reads to improve genome assemblies. Bioinformatics 27, 2957-2963. doi: 10.1093/bioinformatics/btr507

Marchandin, H., Teyssier, C., Campos, J., Jean-Pierre, H., Roger, F., Gay, B., et al. (2010). Negativicoccus succinicivorans gen. nov., sp. nov., isolated from human clinical samples, emended description of the family Veillonellaceae and description of Negativicutes classis nov., Selenomonadales ord. nov. and Acidaminococcaceae fam. nov. in the bacterial phylum Firmicutes. Int. J. Syst. Evol. Microbiol. 60, 1271-1279. doi: 10.1099/ijs.0.013102-0 
Mitra, S., Stärk, M., and Huson, D. H. (2011). Analysis of 16S rRNA environmental sequences using MEGAN. BMC Genomics 12(Suppl. 3):S17. doi: 10.1186/14712164-12-S3-S17

Oberhardt, M. A., Zarecki, R., Gronow, S., Lang, E., Klenk, H. P., Gophna, U., et al. (2015). Harnessing the landscape of microbial culture media to predict new organism-media pairings. Nat. Commun. 6:8493. doi: 10.1038/ ncomms 9493

Reichardt, N., Duncan, S. H., Young, P., Belenguer, A., McWilliam Leitch, C., Scott, K. P., et al. (2014). Phylogenetic distribution of three pathways for propionate production within the human gut microbiota. ISME J. 8, 1323-1335. doi: 10.1038 /ismej.2014.14

Rose, T. M., Henikoff, J. G., and Henikoff, S. (2003). CODEHOP (COnsensusDEgenerate hybrid oligonucleotide primer) PCR primer design. Nucleic Acids Res. 31, 3763-3766. doi: 10.1093/nar/gkg524

Shen, Y. F. (2011). Handbook of Chinese liquor making technology. 1st edn. Beijing, China: China Light Industry Press. (in Chinese).

Song, L., and Dong, X. (2008). Clostridium amylolyticum sp. nov., isolated from $\mathrm{H}_{2}$-producing UASB granules. Int. J. Syst. Evol. Microbiol. 58, 2132-2135. doi: $10.1099 /$ ijs.0.65635-0

Tao, Y., Li, J., Rui, J., Xu, Z., Zhou, Y., Hu, X., et al. (2014). Prokaryotic communities in pit mud from different-aged cellars used for the production of chinese strong-flavored liquor. Appl. Environ. Microbiol. 80, 2254-2260. doi: 10.1128/AEM.04070-13

Tao, Y., Wang, X., Li, X., Wei, N., Jin, H., Xu, Z., et al. (2017). The functional potential and active populations of the pit mud microbiome for the production of Chinese strong-flavour liquor. Microb. Biotechnol. 10, 1603-1615. doi: 10.1111/1751-7915.12729

Vital, M., Gao, J., Rizzo, M., Harrison, T., and Tiedje, J. M. (2015). Diet is a major factor governing the fecal butyrate-producing community structure across Mammalia, Aves and Reptilia. ISME J. 9, 832-843. doi: 10.1038/ ismej.2014.179

Vital, M., Howe, A. C., and Tiedje, J. M. (2014). Revealing the bacterial butyrate synthesis pathways by analyzing (meta) genomic data. MBio 5, e00889-e00814. doi: 10.1128/mBio.00889-14

Vital, M., Penton, C. R., Wang, Q., Young, V. B., Antonopoulos, D. A., Sogin, M. L., et al. (2013). A gene-targeted approach to investigate the intestinal butyrate-producing bacterial community. Microbiome 1:8. doi: 10.1186/2049-2618-1-8

Wang, C. D., Chen, Q., Wang, Q., Li, C. H., Leng, Y. Y., Li, S. G., et al. (2014). Long-term batch brewing accumulates adaptive microbes, which comprehensively produce more flavorful Chinese liquors. Food Res. Int. 62, 894-901. doi: 10.1016/j.foodres.2014.05.017
Wang, X., Du, H., and Xu, Y. (2017). Source tracking of prokaryotic communities in fermented grain of Chinese strong-flavor liquor. Int. J. Food Microbiol. 244, 27-35. doi: 10.1016/j.ijfoodmicro.2016.12.018

Wang, H. Y., Zhang, X. J., Zhao, L. P., and Xu, Y. (2008). Analysis and comparison of the bacterial community in fermented grains during the fermentation for two different styles of Chinese liquor. J. Ind. Microbiol. Biotechnol. 35, 603-609. doi: 10.1007/s10295-008-0323-Z

Wuyts, S., Van Beeck, W., Oerlemans, E. F., Wittouck, S., Claes, I. J., De Boeck, I., et al. (2018). Carrot juice fermentations as man-made microbial ecosystems dominated by lactic acid bacteria. Appl. Environ. Microbiol. 84, e00134-e00118. doi: 10.1128/AEM.00134-18

Xiao, C., Lu, Z. M., Zhang, X. J., Wang, S. T., Ao, L., Shen, C. H., et al. (2017). Bio-heat is a key environmental driver shaping microbial community of medium-temperature Daqu. Appl. Environ. Microbiol. 83, e01550-e01517. doi: 10.1128/AEM.01550-17

Yoon, S. H., Ha, S. M., Kwon, S., Lim, J., Kim, Y., Seo, H., et al. (2017). Introducing EzBioCloud: a taxonomically united database of $16 \mathrm{~S}$ rRNA and whole genome assemblies. Int. J. Syst. Evol. Microbiol. 67, 1613-1617. doi: 10.1099/ijsem.0.001755

Zhang, J., Fang, F., Chen, J., and Du, G. (2014). The arginine deiminase pathway of koji bacteria is involved in ethyl carbamate precursor production in soy sauce. FEMS Microbiol. Lett. 358, 91-97. doi: 10.1111/1574-6968.12542

Zheng, X. W., and Han, B. Z. (2016). Baijiu (白酒), Chinese liquor: history, classification and manufacture. J. Ethn. Foods 3, 19-25. doi: 10.1016/j.jef.2016.03.001

Zou, W., Ye, G., and Zhang, K. (2018a). Diversity, function, and application of Clostridium in Chinese strong flavor baijiu ecosystem: a review. J. Food Sci. 83, 1193-1199. doi: 10.1111/1750-3841.14134

Zou, W., Zhao, C., and Luo, H. (2018b). Diversity and function of microbial community in Chinese strong-flavor baijiu ecosystem: a review. Front. Microbiol. 9:671. doi: 10.3389/fmicb.2018.00671

Conflict of Interest Statement: The authors declare that the research was conducted in the absence of any commercial or financial relationships that could be construed as a potential conflict of interest.

Copyright (c) 2019 Chai, Lu, Zhang, Ma, Xu, Qian, Xiao, Wang, Shen, Shi and $X u$. This is an open-access article distributed under the terms of the Creative Commons Attribution License (CC BY). The use, distribution or reproduction in other forums is permitted, provided the original author(s) and the copyright owner(s) are credited and that the original publication in this journal is cited, in accordance with accepted academic practice. No use, distribution or reproduction is permitted which does not comply with these terms. 